\title{
Thermography in manufacturing: nondestructive evaluation of ultrasonic spot welds of automotive battery pack
}

\author{
by P. Shpartko*, L. Lev**, N. Kondratyev**, J. Soh** \\ *Dept. of Applied Mathematics, St. Petersburg Polytechnical University, Polytechnicheskaya str. 29, 195251 St. \\ Petersburg, Russia, polina190189@yahoo.com \\ ${ }^{* *}$ GM R\&D and Planning, Mound Road 30500, 48090 Warren MI, USA, leo.lev@gm.com
}

\begin{abstract}
Flash thermography-based nondestructive evaluation (NDE) procedure for inspection of spot welds of automotive battery packs has been developed. A mathematical model of heat propagation has been proposed. The underlying equations have been solved analytically for simple geometry and numerically, using ANSYS FEA package, for the case of general geometry. Based on the proposed model, the size of the fused area of spot welds was determined from thermographic experiments and a correlation with direct measurements was obtained.

In a separate set of experiments, the response to a typical flash impulse was determined and the characteristics of the heat pulse were determined by using least square approximation. The reconstructed shape of the flash impulse was used for NDE of welds between thin sheets.
\end{abstract}

\section{Introduction}

Automotive battery packs are assembled using ultrasonic spot welding. To insure $100 \%$ good welds, a quick and reliable nondestructive evaluation (NDE) technique is required. The requirements for these welds include low electrical resistance and high tensile strength. These requirements are satisfied if the fused area of the welds is of the sufficient size. A thermography-based NDE technique, capable of determining the size of the fused area of the weld has been developed in GM R\&D.

A few other NDE techniques can determine the size of the fused area of a spot weld and assess the weld quality. Such techniques include acoustic microscopy, shearography and X-ray radiometry [1, 2]. However, the NDE technique, capable of providing reliable results in production is still lacking. For example, X-ray radiometry is relatively slow, costly, and requires special personnel protection. Ultrasonic inspection could have only a limited use for battery welds since the battery cannot be immersed in liquid and the presence of coupling agent on the welds is highly undesirable.

Traditionally, thermography-based NDE have been considered as having insufficient resolution and reliability [1, 2]. Present work corrects this perception. The developed flash thermography-based NDE procedure is shown to provide high precision, reliable data, correlated with mechanical measurements. Developed for defect detection [3-7], this technique is fast, noncontact and relatively inexpensive. Current report communicates our results and mathematical underpinnings of the method.

\section{Experimental Details}

Samples with a single lap weld were prepared by ultrasonically welding sheets of copper with thickness of $0.2 \mathrm{~mm}$ and $0.9 \mathrm{~mm}$.

Flash thermography experiments were conducted by using an Elinchrom RX1200 studio strobe flash lamp with energy of up to 1200 joules per flash. The light was flashed onto the front surface of the sample. The duration of the flash was nominally $1 / 1450$ of a second. The welded samples were positioned between the flash lamp and an infra-red (IR) camera (figure 1). A FLIR IR camera SC4000 with Indium Antimonite detector (1.5-5.0 $\mu \mathrm{m}$ spectral range) was focused onto the back (opposite to the illuminated) surface of the sample and was synchronized with the flash lamp. The data was collected at sampling rates of up to $2,000 \mathrm{~Hz}$. To increase both absorptivity and emissivity of specimens they were coated with a thin layer of Krylon flat white paint \#1502 (emissivity 0.989 at the wavelength of $10 \mu$ and 0.992 at wavelength of $3 \mu$ ), followed by a coating of Krylon flat black \#51602 (emissivity 0.956 at $10 \mu$ wavelength and 0.95 at $5 \mu$ wavelength).

The intensity and duration of the light flash were calculated from flash thermography experiments with a thin copper plate substituted in place of a sample. Since the properties of copper are well known, the temporal shape of the heat flash was reconstructed by solving inverse heat propagation problem.

Electrical resistance and the strength of the samples were measured independently and correlated with the results of thermography expermiments. 


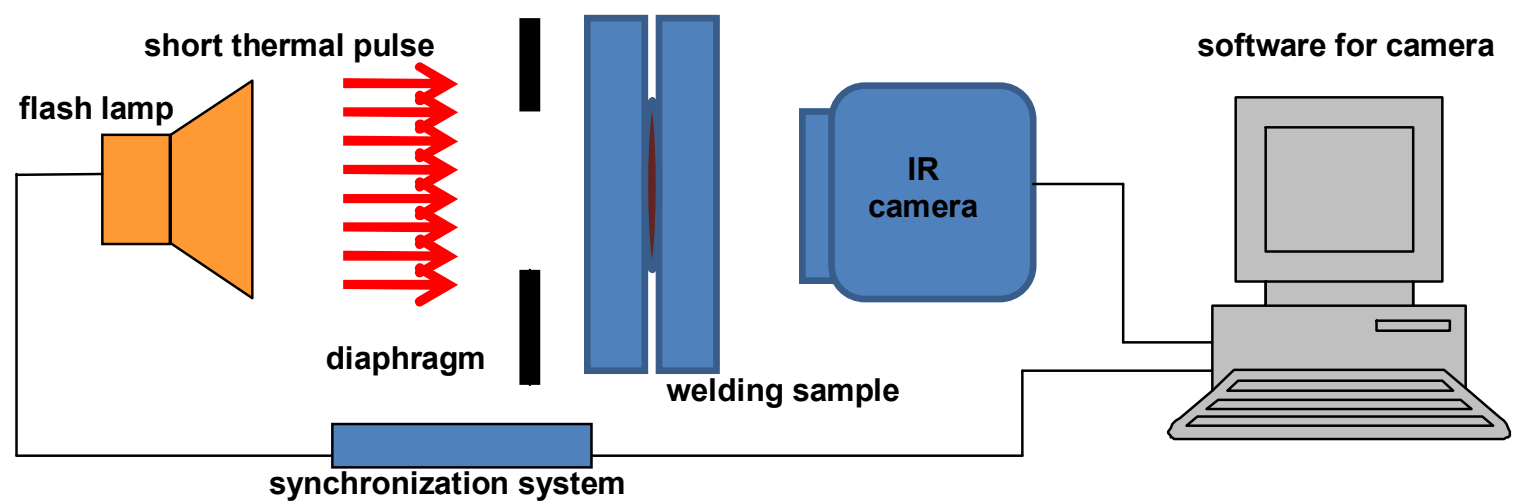

Fig. 1. Experimental setup

\section{Experimental results and discussion}

It has been determined that a thin air gap separated the welded sheets of the lap-welded samples everywhere except in the fused area, resulting in substantial difference in the rates of heat propagation through the fused and nonfused area. Therefore, the temperature rise in the points on the surface of the sample, lying over fused area was expected to be substantially more rapid than in the points lying above a non-fused area.

This was confirmed in the experiments, as illustrated in figures 2 and 3, presenting a thermographic image of the backside of a specimen as measured by the IR camera (figures 2, a, and 3, a). Brighter (red and yellow) colors correspond to higher temperatures. The temperature histories of three characteristic points are shown in figure 2 , b. The temperature derivatives of these three points are presented in figure $3, \mathrm{~b}$. As can be seen from the figures $2, \mathrm{~b}$ and $3, \mathrm{~b}$, the temperature rise is more rapid in the points lying over the fused area. By detecting all the areas with high rate of temperature rise, the total fused area of the weld can be measured. To establish the precision of the correlation between the areas with high temperature rise rates and the fused areas, a mathematical model was used.

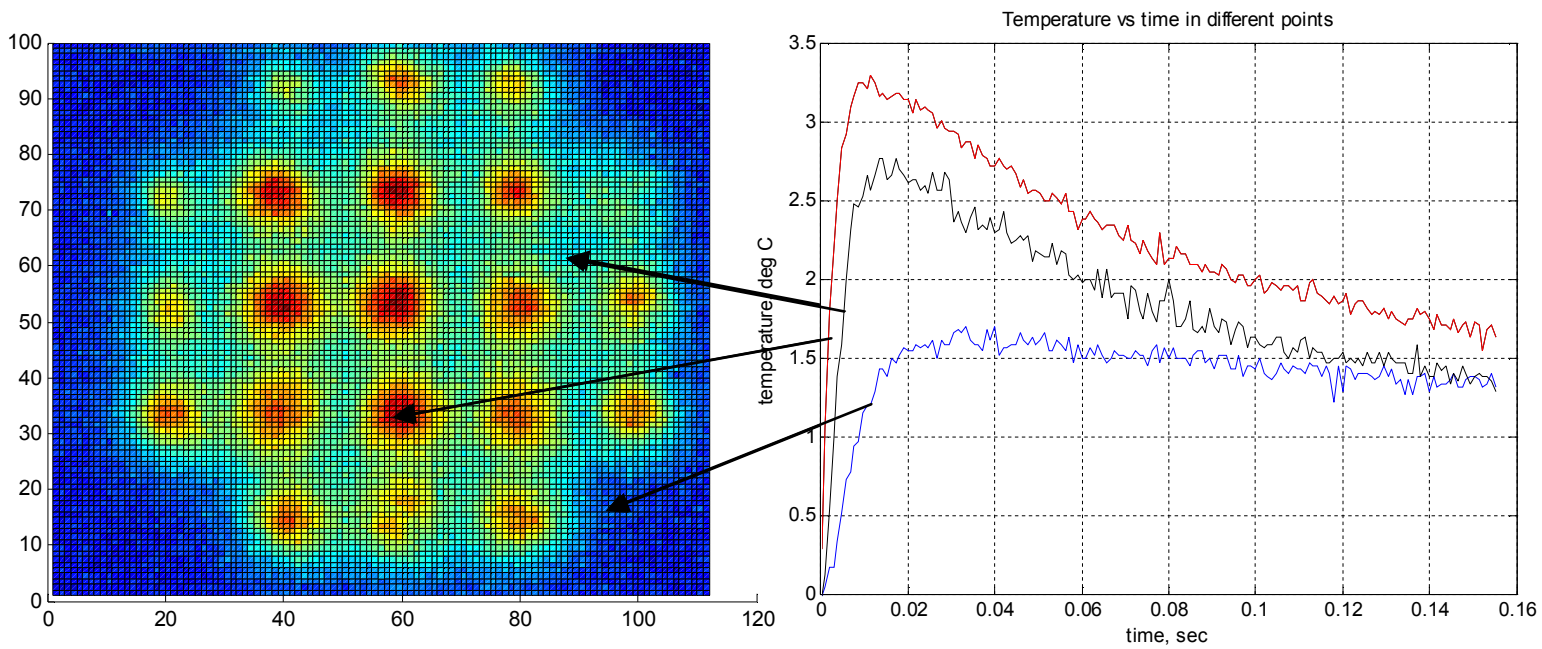

(a)

(b)

Fig. 2. Temperature vs time curves in different points on back surface of weld sample 


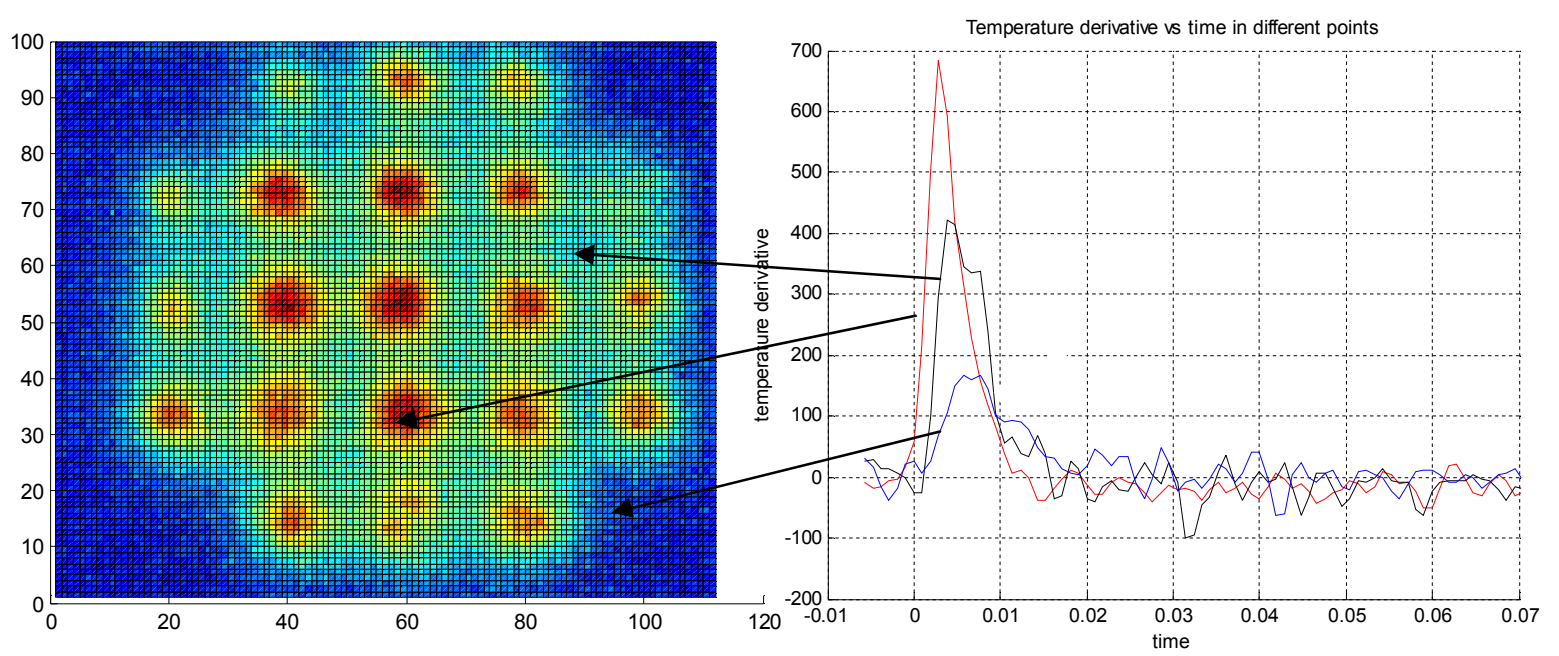

(a)

(b)

Fig. 3. Temperature derivative vs time curves in different points on back surface of weld sample

\section{Mathematical formulation and developed procedure}

\subsection{Mathematical formulation}

During the flash thermography test the energy, emitted by flash lamp, is absorbed by the front surface of the specimen and is converted to heat. Heat propagates through the interior of the sample and is detected by the thermal camera as the temperature rise on the opposite surface.

To simplify mathematical model we made the following assumptions:

- No heat losses,

- The front surface of the sample is flat,

- The energy distribution of the original pulse is uniform across the sample surface and

- The power of the lamp is constant during the pulse.

Heat propagation in the sample is governed by the parabolic partial differential equation and, together with initial and boundary conditions, represents an initial boundary value problem for temperature distribution $T(x, t)$. Denoting thermal diffusivity, thermal conductivity and density as $a, k, \rho$, duration and intensity of thermal pulse as $t_{0}$ and $q$ and the part of the surface illuminated by flash as $\Gamma_{1}$ and not illuminated as $\Gamma_{2}$, we obtained the following formulation:

$$
\left\{\begin{array}{l}
\frac{\partial T}{\partial t}=a \Delta T \quad(a=k / \rho c) \\
\left.T\right|_{t=0}=0 \\
\left.\frac{\partial T}{\partial n}\right|_{\Gamma_{1}}= \begin{cases}q / k, & 0 \leq t \leq t_{0} \\
0, & t_{0} \leq t\end{cases} \\
\left.\frac{\partial T}{\partial n}\right|_{\Gamma_{2}}=0
\end{array}\right.
$$

In our experiments, temperature history in each point on the surface of the sample was recorded by the IR camera as a discrete set of data:

$$
T_{l}\left(t_{n}, x_{i}, y_{j}\right), \quad n=\overline{1, N}, \quad i=\overline{1, M_{1}}, \quad j=\overline{1, M_{2}}
$$


where $T_{l}$ is the temperature of the surface of the sample. The problem was to find the fused area of the sample using the data provided in Eq. (2).

\subsection{Evaluation of the flash intensity and duration}

Precise knowledge of the pulse duration $t_{0}$ and its intensity $q$ is required to solve Eq. (1). They were measured in the series of separate tests, in which a thin copper plate was substituted in place of sample (figure 1). In this case, the Eq. (1) takes the form of 1D problem and can be solved analytically. If the thickness of the plate is $I$, for the points on the backside of the flat plate the analytical solution is:

$$
T(l, t)= \begin{cases}\frac{q}{k}\left(-\frac{l}{6}+\frac{a}{l} t-\frac{2 l}{\pi^{2}} \sum_{n=1}^{\infty} \frac{(-1)^{n}}{n^{2}} \exp \left(-\frac{\pi^{2} n^{2}}{l^{2}} a t\right)\right), \quad 0 \leq t \leq t_{0} \\ \frac{q}{k}\left(\frac{a}{l} t_{0}-\frac{2 l}{\pi^{2}} \sum_{n=1}^{\infty} \frac{(-1)^{n}}{n^{2}} \exp \left(-\frac{\pi^{2} n^{2}}{l^{2}} a t\right)\left(1-\exp \left(\frac{\pi^{2} n^{2}}{l^{2}} a t_{0}\right)\right), \quad t_{0} \leq t\right.\end{cases}
$$

The experimental results of the temperature rise for the case of flat copper plate are shown in figure 4 . The sampling rate of the thermal camera was approximately $1000 \mathrm{~Hz}$. The temperature rise within the first $20 \mathrm{msec}$ is an artifact of the test, caused by direct reflection of the light into the lens of the IR camera. The values of $t_{0}$ and $q$ were obtained as parameters by fitting the solution Eq. (3) to the experimental results using least squares approximation.

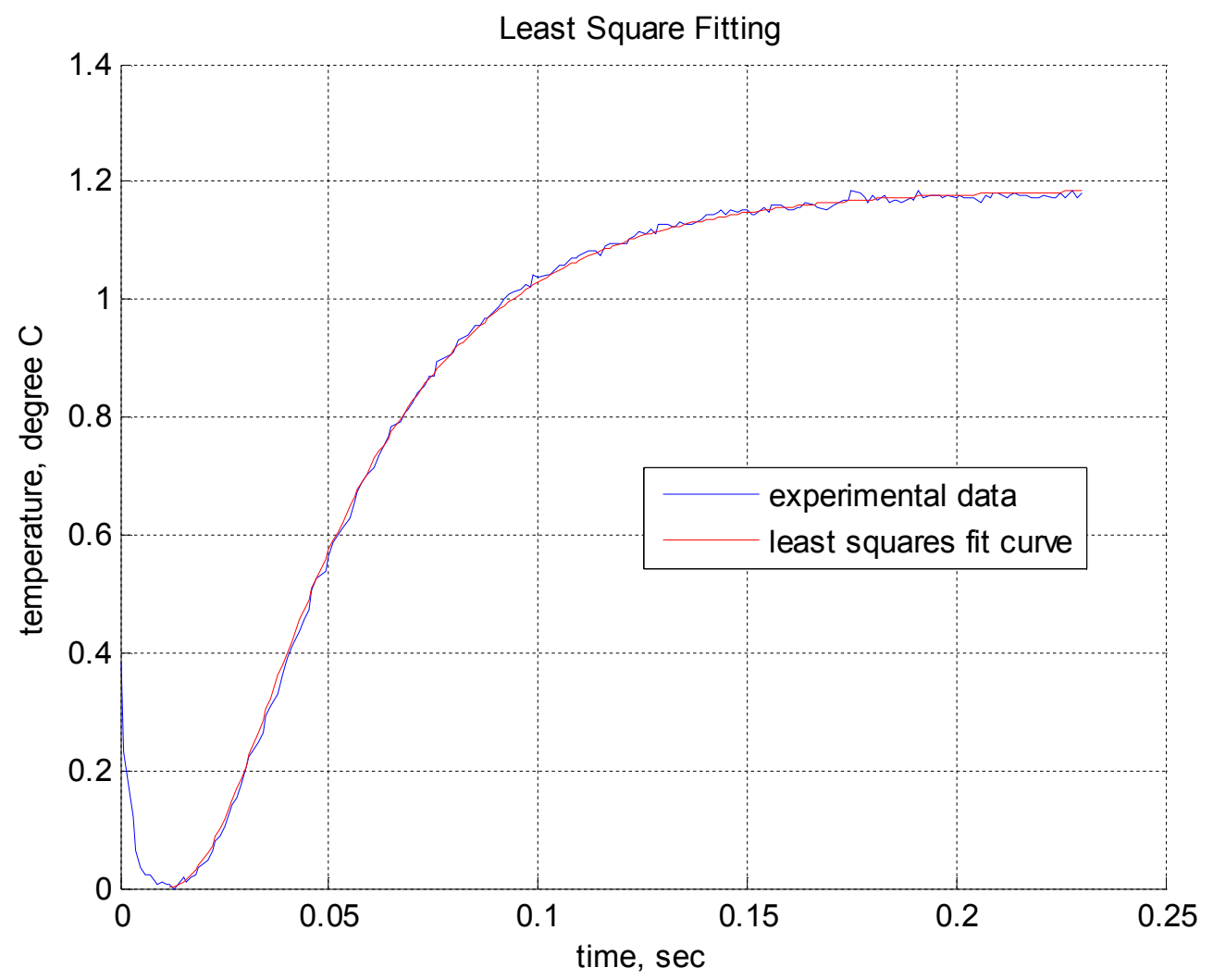

Fig. 4. Flash characteristics evaluation by least squares fit procedure

\subsection{Modeling of heat propagation using FEA package}

Eq. (1) was solved numerically using ANSYS FEA package. Two configurations of fused area were considered:

1. One contiguous fused area in the weld spot.

2. Multiple small fused areas. 


\subsubsection{Single fused area}

The cross section of the weld is shown in figure 5 , a. The calculated temperature histories for different points on the back surface of the sample are shown in figure $5, \mathrm{~b}$. As can be seen from the figure $5, \mathrm{~b}$, the rate of temperature rise is substantially lower for points located not over the projection of the fused area.

To illustrate this point, numerical derivatives of the temperature histories were calculated for these three points (figure 6). The values of the derivative for "center" and "border" points are 2 times greater than the ones for the points out of the projection of the fused area. Numerical experiments show that this difference does not depend on the size of the fused area when the latter changes in the range of between the whole weld spot to $1 / 4$ of its size.

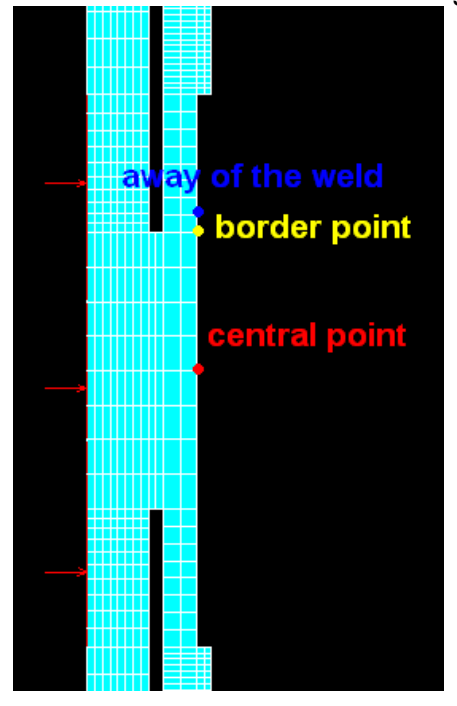

(a)

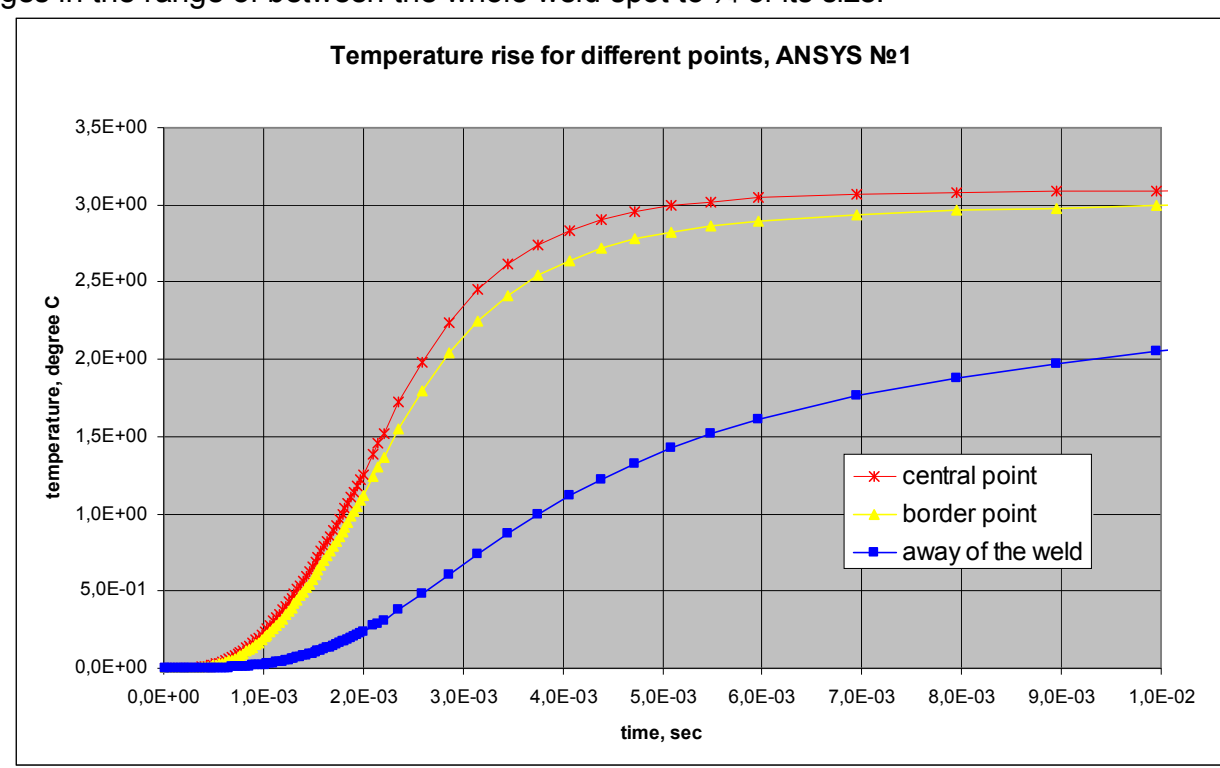

(b)

Fig. 5. Numerical model with single fused area

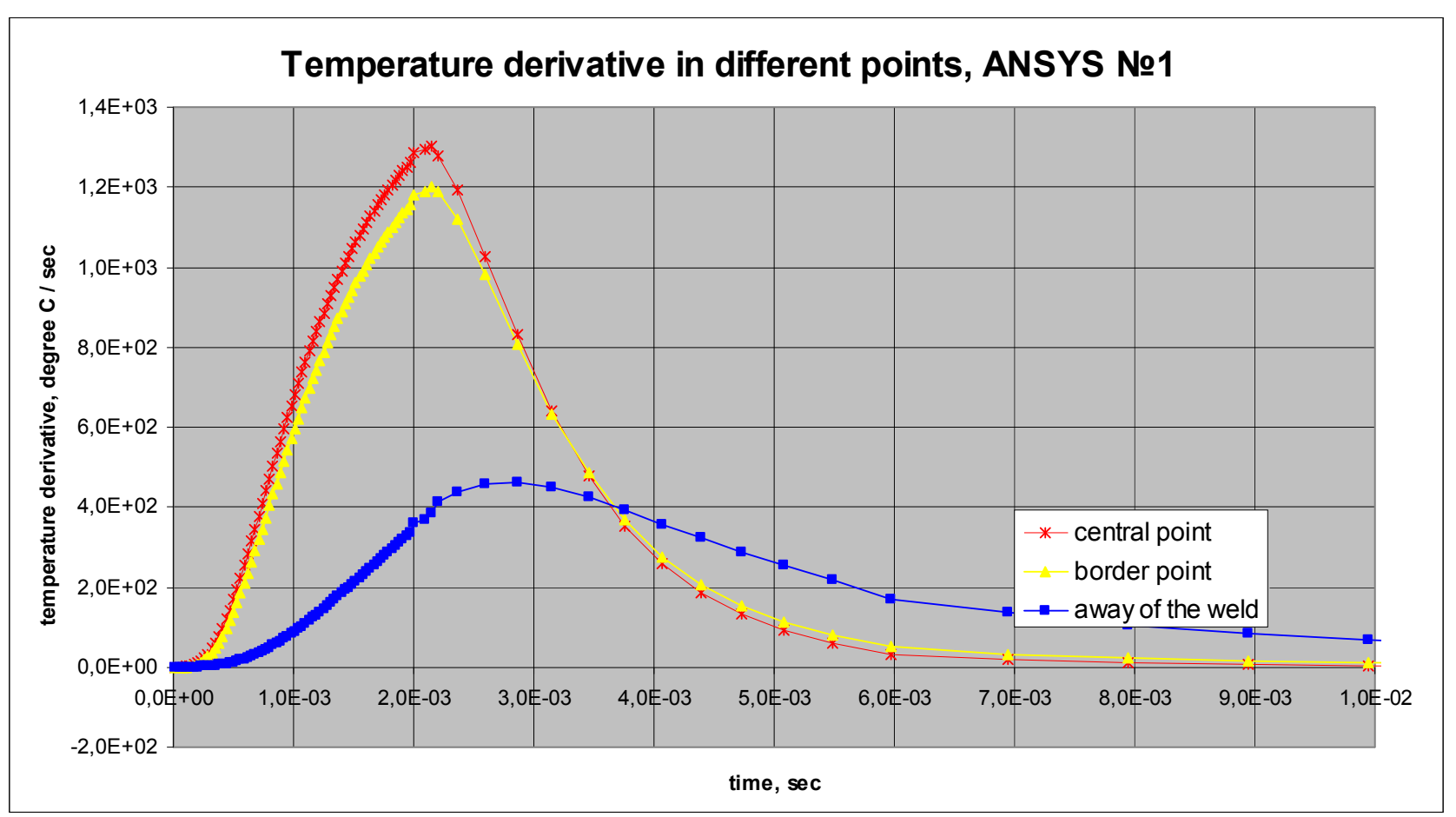

Fig. 6. Numerical model with single fused area: temperature derivative vs time curves 


\subsubsection{Multiple fused areas}

The view of the fused surface of the lap weld after it was tested destructively is shown in figure 7, a. The weld was formed with the tool equipped with course knurling that resulted in multiple fused areas. This geometry was modeled and the resulting temperature distribution was shown to be a better approximation for some of the experimental data.

Figure 7, b, shows the temperature histories for different points of sample's back side. These points are schematically marked on the left of figure 7. Here fused areas denoted by "spots" and air gapes between "spots" are denoted as "holes".

While the difference in temperature rise rates (see figure 8 ) is not as large as in the single spot model, the projection of the fused area on the surface of the sample still can be identified.

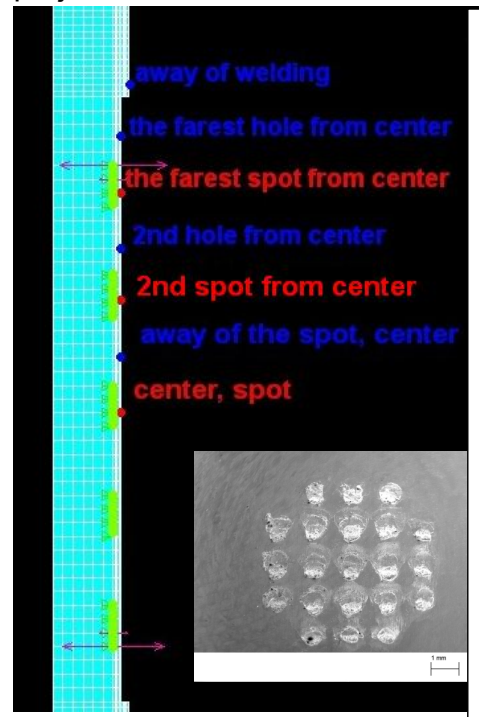

(a)
Temperature for different points, ANSYS model №2

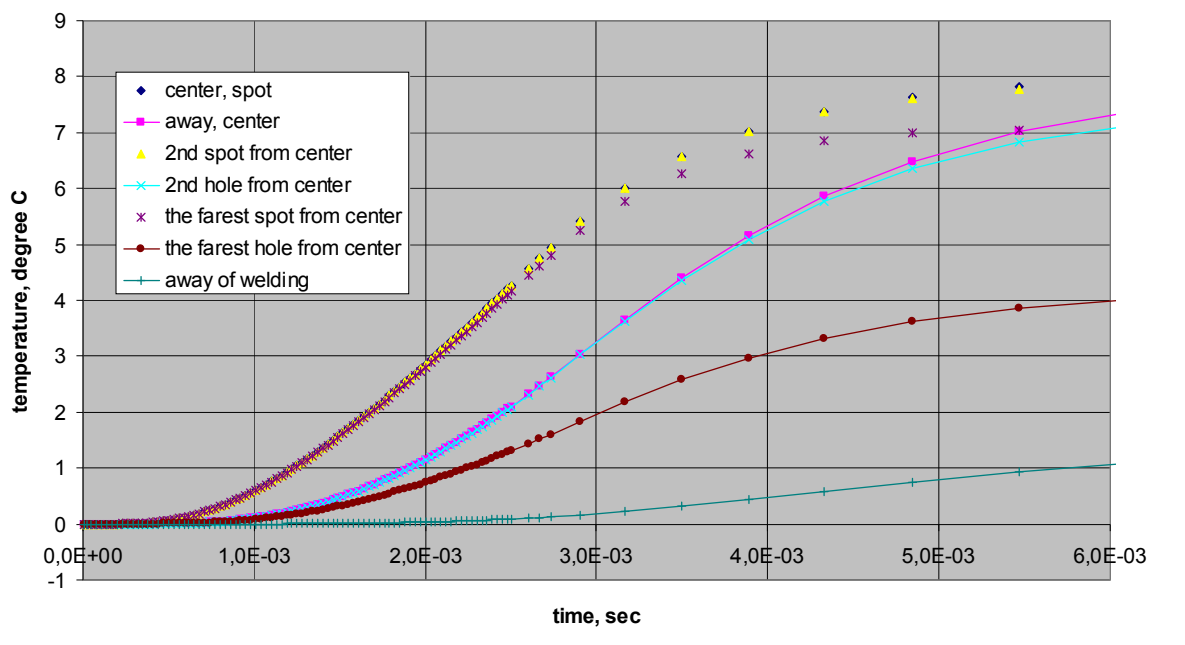

(b)

Fig. 7. Numerical model with multiple fused areas

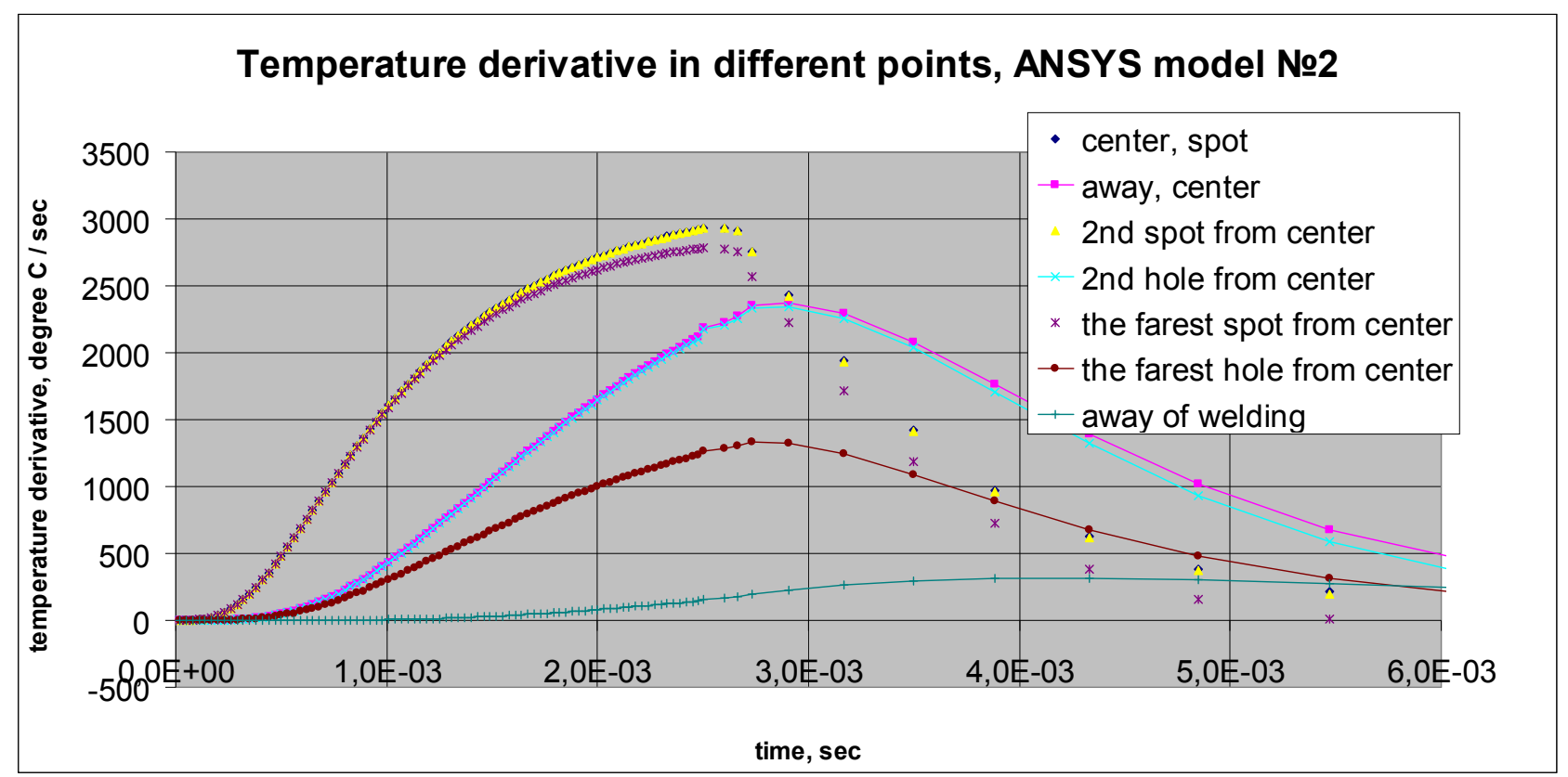

Fig. 8. Numerical model with multiple fused areas: temperature derivative vs time curves 


\subsection{Evaluation of the weld fused area}

Figure 9 shows normalized experimental and calculated temperature histories. The graph contains a pair of "hot" and "cold" curves from numerical model and experiments.

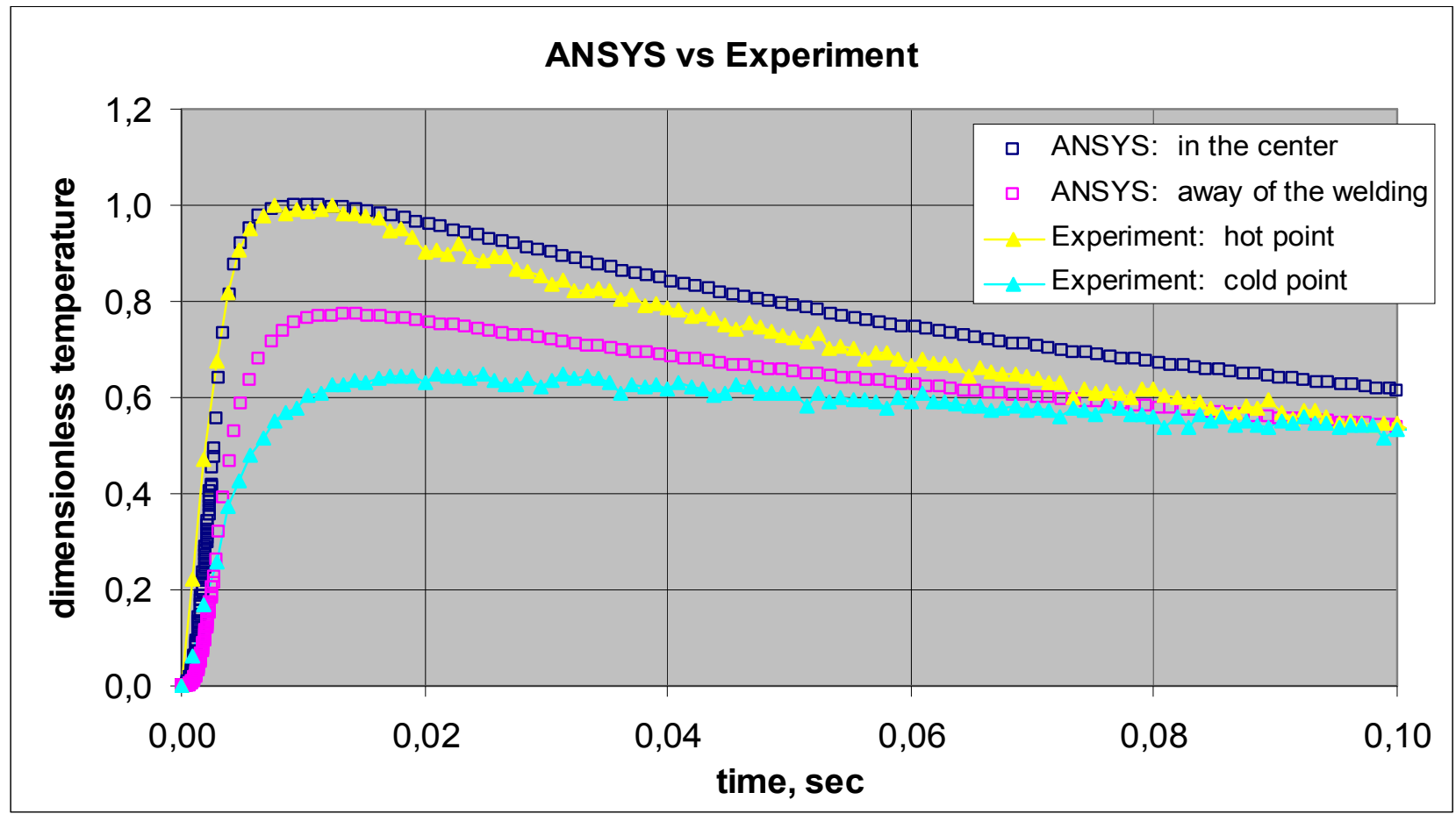

Fig. 9. Comparison of numerical and experimental data dimensionless

The derivative of the temperature history in the point on the surface of the sample was taken as an indication of the presence of fused area underneath. By combining these points, the entire fused area was reconstructed nondestructively and its size was estimated.

\section{Correlation with strength measurements}

25 samples were produced by ultrasonic welding and their fused areas were measured in flash experiments. Their electrical resistance of the samples was measured as well using special fixture. Thermographical data was processed by low pass filter and the fused areas of samples were evaluated nondestructively and compared with electrical conductivity (see figure 10). As can be seen from the figure 10, the results of both measurements exhibit correlation, as was expected.

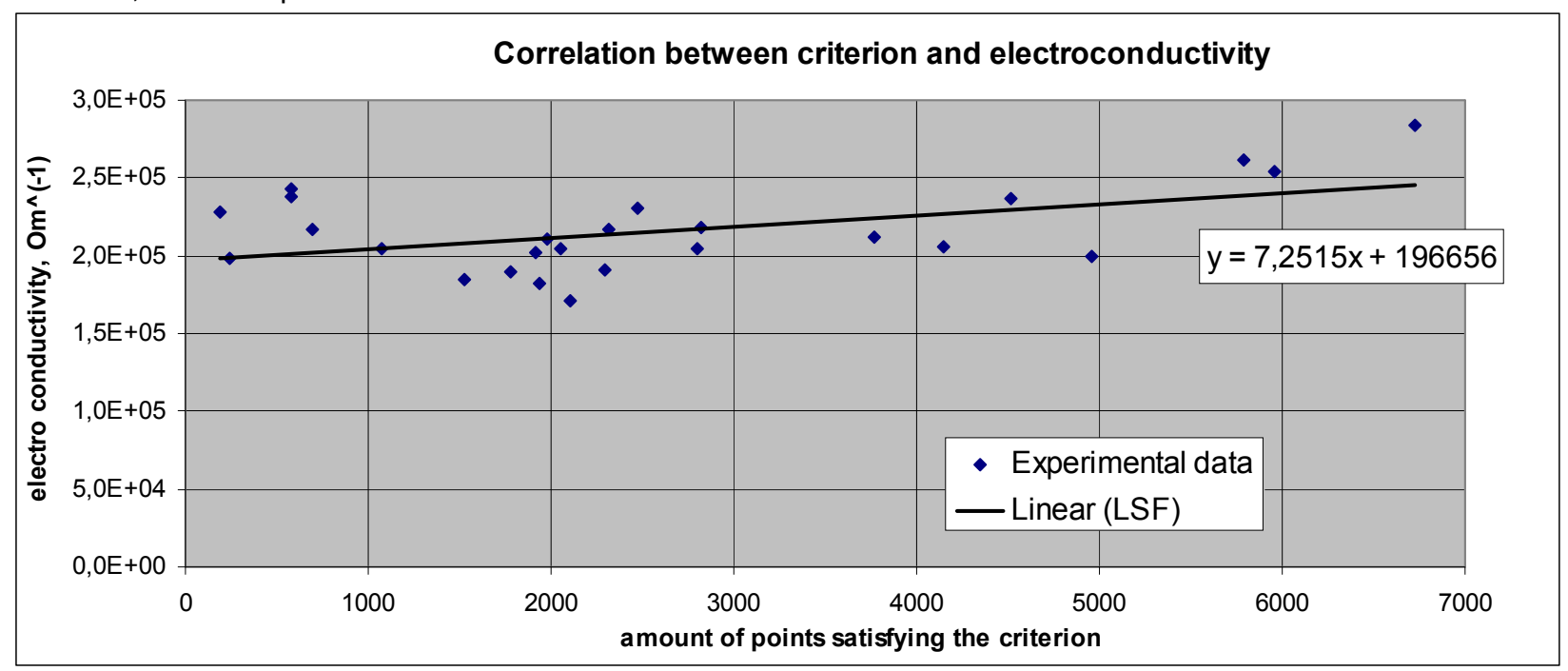

Fig. 10. Electrical conductivity of the welds vs. the size of the fused area of the weld 


\section{REFERENCES}

[1] F. Chen, E.Y. Kuo, P.M. Wang, C.T. Griffen, "Nondestructive Evaluation of Spot Weld Integrity/Quality: Method Comparison". Proceedings of SAE World Congress, SAE Technical paper 1999-01-0944.

[2] D. Turler, D. Hopkins, F. Reverdy, "Nondestructive evaluation of spot welds using acoustic and thermographic imaging techniques", Proceedings of SAE World Congress, SAE Technical paper 2003-01-0980.

[3] S.K. Lau, D.P. Almond, J.M. Milne, "A quantitative analysis of pulsed video thermography". NDT \& E International, vol. 24, pp. 195-202, 1991.

[4] V. Vavilov, X. Maldague, B. Dufort, F. Robitaille, J. Picard, "Thermal nondestructive testing of carbon epoxy composites: detailed analysis and date processing". NDT \& E International, vol. 26, pp. 85-95, 1993.

[5] V. Vavilov, E. Grinzato, P.G. Bison, S. Marinetti, M. J. Bales, "Surface transient temperature inversion for hidden corrosion characterisation: theory and application”, Int. J. Heat Mass Transfer, vol. 2, pp. 355-371. 1996.

[6] M.A. Omar, Y. Zhou, A. Mayyas, A. Qattawi, "Pulse thermography for inspecting Automative Components and Materials". Proceedings of SAE World Congress, SAE Technical paper 2010-01-0959.

[7] V. Vavilov, "Thermal nondestructive testing: short history and state-of-art". Proceedings of 1st Quantitative InfraRed Thermography conference, QIRT92 - Eurotherm Series 27 - EETI ed., Paris 1992. 